\title{
Competitive and core allocations in large economies with differential information ${ }^{\star}$
}

\section{Ezra Einy $^{1}$, Diego Moreno ${ }^{2}$, and Benyamin Shitovitz ${ }^{3}$}

1 Department of Economics, Ben Gurion University of Negev, Beer Sheva, 841105, ISRAEL (e-mail: einy@bgumail bgu ac il);

2 Departamento de Economía, Universidad Carlos III de Madrid, 28903 Getafe, SPAIN (e-mail: dmoreno@eco uc3m es);

3 Department of Economics, University of Haifa, Haifa, 31905, ISRAEL (e-mail: binya@econ haifa ac il)

Received March 22, 2000; revised version: May 1, 2000

Summary. We study the core and competitive allocations in exchange economies with a continuum of traders and differential information. We show that if the economy is "irreducible", then a competitive equilibrium, in the sense of Radner (1968, 1982), exists. Moreover, the set of competitive equilibrium allocations coincides with the "private core" (Yannelis, 1991). We also show that the "weak fine core" of an economy coincides with the set of competitive allocations of an associated symmetric information economy in which the traders information is the joint information of all the traders in the original economy.

Keywords and Phrases: General equilibrium in large economies with differential information, Private core, Weak fine core.

JEL Classification Numbers: D50, D82.

\section{Introduction}

The purpose of this paper is to study the properties of core and competitive allocations in large exchange economies with differential information. Radner (1968

* This work was done while Einy and Shitovitz visited the Department of Economics of the Universidad Carlos III de Madrid Einy acknowledges the financial support of the Universidad Carlos III de Madrid Moreno acknowledges the support of the Spanish Ministry of Education, grant PB970091 Shitovitz acknowledges the support of the Spanish Ministry of Education, grant SAB98-0059 Correspondence to: D Moreno 
and 1982) introduces a model of an exchange economy with differential information in which every trader is characterized by a state dependent utility function, a random initial endowment, an information partition, and a prior belief. In this framework, traders arrange contingent contracts for trading commodities before they obtain any information about the realized state of nature. Radner (1968) extends the notion of Arrow-Debreu competitive equilibrium to this model. In the definition of competitive equilibrium (in the sense of Radner), the information of an agent places a restriction on his feasible trades (i.e., his budget set): better information allows for more contingent trades (i.e., enlarges the agent's budget set). Thus, in a Radner competitive equilibrium better informed agents are generally, ceteris paribus, better off (and they are never worse off) than those with worse information; i.e., a competitive equilibrium rewards the information advantage of a trader.

We consider two period Radner-type economies with a finite number of states of nature and a continuum of traders. In these economies there is uncertainty about the state of nature. In the first period traders arrange contracts that may be contingent on the realized state of nature in the second period. Consumption takes place in the second period. We provide a straightforward extension to these economies of the notion of Radner competitive equilibrium, and examine conditions that guarantee its existence. Also we study the set of core allocations in these economies. It is well known that in perfectly competitive economies (that is, when no individual can affect the overall outcome) with complete information the core coincides with the set of competitive allocations (see, e.g., Aumann, 1964). In an economy with differential information, the set of allocations that a coalition can block depends upon the initial information and the communication opportunities of the members of the coalition. Thus, several alternative notions of core can be considered.

Yannelis (1991) introduces the notion of private core, and shows that under appropriate assumptions the private core of an economy is non-empty. In the private core the set of feasible allocations for a blocking coalition must involve a net trade of each member of the coalition that is measurable with respect to his information partition. The private core has some interesting properties: Koutsougeras and Yannelis (1993) show that if there is a finite number of traders, the private core is coalitionally incentive compatible-see Section 4 in Koutsougeras and Yannelis (1993). Also the private core rewards the information advantage of a trader-see Section 5 in Koutsougeras and Yannelis (1993), and the discussion below.

Koutsougeras and Yannelis (1993), see also Allen (1991), introduce the notion of weak fine core, a version of Wilson's (1978) fine core, and showed that this core is non-empty. In the definition of the weak fine core blocking net trades are measurable with respect to the joint partition of all the members of the coalition, but in addition all net trades are measurable with respect to the joint partition of all the traders.

In our context, the private core is the appropriate notion of core when the traders have no access to any communication system, and therefore cannot ex- 
change information. The weak fine core is the appropriate notion of core when the traders have access to a communication system that allows them to fully share their information, and under the maintained assumption of perfect competition (that is, assuming that individuals are also "small" from the point of view of information). We study the relations of these cores and the set of competitive allocations in the economies described above, with a continuum of traders and differential information.

First we establish conditions under which a Radner competitive equilibrium exists: we show that if an economy is irreducible, and if the traders' utility functions are continuous and increasing, then an equilibrium exists. The existence of competitive equilibrium in economies with a continuum of traders and complete information was studied in Aumann (1966) and Hildenbrand (1970). We establish existence of equilibrium using general results from Hildenbrand (1974). The irreducibility condition was introduced by Mckenzie (1959) for exchange economies with a finite number of traders, and it has been extended to economies with a continuum of traders by Hildenbrand (1974). It expresses the idea that the endowment of every coalition, if added to the allocation of the complementary coalition, can be used to improve the welfare of every member of the complementary coalition. We show that an economy is irreducible if, for example, the initial endowment of every trader is strictly positive at each state of nature.

Next we show that if an economy is irreducible, then the private core of the economy coincides with the set of Radner competitive equilibrium allocations. Thus, private core allocations reward the information advantage of a trader. We provide simple examples which show that this result may not hold for reducible economies. For the weak fine core, we show that under mild assumptions it coincides with the set of competitive allocations of an associated symmetric information economy. This associated economy is identical to the original economy, except for the traders' information, which is the joint information of all the traders in the original economy. Specifically, this result holds if the traders' utility functions are continuous and strictly increasing, and if for every trader there is a state of nature at which his initial endowment is non-zero. Moreover, the result holds whether or not the economy is irreducible.

Thus, whereas private core allocations reward the information advantage of a trader, when the possibility of sharing information is introduced the information advantage is worthless; e.g., if two traders A and B have identical characteristics, except that $\mathrm{A}$ is better informed than B (i.e., A's information partition is finer than B's) then in a private core allocation trader A may be better off than trader B; in a weak fine core allocation, however, both traders are equally well off (because a weak fine core allocation is a competitive allocation of the associated symmetric information economy). (In sharp contrasts to this result, Einy, Moreno and Shitovitz (1999) have shown that the weak fine bargaining set contains allocations that are not competitive in the associated symmetric information economy, which suggests that the weak fine bargaining set discriminates in favor of better informed agents.) 
The paper is organized as follows. In Section 2 we describe the model. In Section 3 we discuss the existence of competitive equilibrium (in the sense of Radner). In Section 4 we prove the equivalence between competitive and private core allocations. Finally, in Section 5 we establish the equivalence of the weak fine core and the set of competitive allocations of the associated symmetric information economy.

\section{The model}

We consider a Radner-type exchange economy $\mathscr{E}$ with differential information (e.g., Radner, 1968, 1982). The commodity space is $\Re_{+}^{l}$. The space of traders is a measure space $(T, \Sigma, \mu)$, where $T$ is a set (the set of traders), $\Sigma$ is a $\sigma$-field of subsets of $T$ (the set of coalitions), and $\mu$ is a non-atomic measure on $\Sigma$. The economy extends over two time periods, $\tau=0,1$. Consumption takes place at $\tau=1$. At $\tau=0$ there is uncertainty over the state of nature; in this period traders arrange contracts that may be contingent on the realized state of nature at $\tau=1$. There is a finite space of states of nature, denoted by $\Omega$. At $\tau=1$ traders do not necessarily know which state of nature $\omega \in \Omega$ actually occurred, although they know their own endowments, and may also have some additional information about the state of nature. We do not assume, however, that traders know their utility function (see below).

The information of a trader $t \in T$ is described by a partition $\Pi_{t}$ of $\Omega$. We denote by $\mathscr{F}_{t}$ the field generated by $\Pi_{t}$. If $\omega$ is the true state of the economy at $\tau=1$, trader $t$ observes the member of $\Pi_{t}$ which contains $\omega$. Every trader $t \in T$ has a probability measure $q_{t}$ on $\Omega$ which represents his prior beliefs. The preferences of a trader $t \in T$ are represented by a state dependent utility function, $u_{t}: \Omega \times \Re_{+}^{l} \rightarrow \Re$ such that for every $(t, x) \in T \times \Re_{+}^{l}$, the mapping $(t, x) \rightarrow u_{t}(\omega, x)$ is $\Sigma \times \mathscr{B}$ measurable, where $\omega$ is a fixed member of $\Omega$, and $\mathscr{B}$ is the $\sigma$-field of Borel subsets of $\Re_{+}^{l}$. If $x$ is a random bundle (i.e., a function from $\Omega$ to $\Re_{+}^{l}$ ) we denote by $h_{t}(x)$ the expected utility from $x$ of trader $t \in T$. That is

$$
h_{t}(x)=\sum_{\omega \in \Omega} q_{t}(\omega) u_{t}(\omega, x(\omega)) .
$$

An assignment is a function $\mathbf{x}: T \times \Omega \rightarrow \Re_{+}^{l}$ such that for every $\omega \in \Omega$ the function $\mathbf{x}(\cdot, \omega)$ is $\mu$-integrable on $T$. There is a fixed initial assignment $\mathbf{e}$; $\mathbf{e}(t, \omega)$ represents the initial endowment density of trader $t \in T$ in the state of nature $\omega \in \Omega$. We assume that for almost every $t \in T$ the function $\mathbf{e}(t, \cdot)$ is $\mathscr{F}_{t}$-measurable.

Throughout the paper we use the following notations. For two vectors $x=$ $\left(x_{1}, \ldots, x_{l}\right)$ and $y=\left(y_{1}, \ldots, y_{l}\right)$ in $\Re^{l}$ we write $x \geq y$ when $x_{k} \geq y_{k}$ for all $1 \leq k \leq l, x>y$ when $x \geq y$ and $x \neq y$, and $x \gg y$ when $x_{k}>y_{k}$ for all $1 \leq k \leq l$. 


\section{Competitive equilibrium}

In this section we extend Radner's (1982) definition of competitive equilibrium to our model (see Radner, 1982, Section 3.4), and discuss conditions under which its existence can be guaranteed. Throughout the rest of the paper, an economy $\mathscr{E}$ is an atomless economy with differential information as described in Section 2.

A private allocation for an economy $\mathscr{E}$ is an assignment $\mathbf{x}$ such that

(3.1) for almost all $t \in T$ the function $\mathbf{x}(t, \cdot)$ is $\mathscr{T}_{t}$-measurable, and (3.2) $\int_{T} \mathbf{x}(t, \omega) d \mu \leq \int_{T} \mathbf{e}(t, \omega) d \mu$ for all $\omega \in \Omega$.

A price system is a non-zero function $p: \Omega \rightarrow \Re_{+}^{l}$. Let $t \in T$ and let $M_{t}$ be the set of all $\mathscr{F}_{t}$-measurable functions from $\Omega$ to $\Re_{+}^{l}$. For a price system $p$, define the budget set of $t$ by

$$
B_{t}(p)=\left\{x \mid x \in M_{t} \text { and } \sum_{\omega \in \Omega} p(\omega) \cdot x(\omega) \leq \sum_{\omega \in \Omega} p(\omega) \cdot \mathbf{e}(t, \omega)\right\} .
$$

A competitive equilibrium (in the sense of Radner) for an economy $\mathscr{E}$ is a pair $(p, \mathbf{x})$ where $p$ is a price system and $\mathbf{x}$ is private allocation such that

(3.3) for almost all $t \in T$ the function $\mathbf{x}(t, \cdot)$ maximizes $h_{t}$ on $B_{t}(p)$, and

(3.4) $\sum_{\omega \in \Omega} p(\omega) \cdot \int_{T} \mathbf{x}(t, \omega) d \mu=\sum_{\omega \in \Omega} p(\omega) \cdot \int_{T} \mathbf{e}(t, \omega) d \mu$.

A competitive allocation is a private allocation $\mathbf{x}$ for which there exists a price system $p$ such that $(p, \mathbf{x})$ is a competitive equilibrium.

In the literature, condition (3.2) in the definition of a private allocation is written usually with (strict) equality; see, e.g., Radner (1968), Krasa and Yannelis (1994), Allen (1997). Here we follow Radner (1982) who noted that the total amount to be disposed of might not be measurable with respect to the information partition of a single agent. Einy and Shitovitz (1999) provide an example of an economy with differential information which has a competitive equilibrium, but if the inequality (3.2) in the definition of a private allocation is replaced with an equality, then the economy does not have a competitive equilibrium where all prices are non-negative-see Example 2.1 in Einy and Shitovitz (1999). Condition (3.4) ensures that in a competitive equilibrium if a commodity is in excess supply its price is zero. This condition is redundant as it is implied by Walras' Law, which is satisfied in our framework. Nevertheless we include it to facilitate comparison to Radner's (1982) definition.

A function $u: \Re_{+}^{l} \rightarrow \Re$ is (strictly) increasing if for all $x, y \in \Re_{+}^{l},(x>y)$ $x \gg y$ implies $u(x)>u(y)$.

Throughout the paper we will often refer to the following conditions.

(A.1) For every $\omega \in \Omega$ we have $\int_{T} \mathbf{e}(t, \omega) d \mu \gg 0$.

(A.2) For almost all $t \in T$ and for every $\omega \in \Omega$, the function $u_{t}(\omega, \cdot)$ is continuous and increasing on $\Re_{+}^{l}$. 
(A.3) Irreducibility: for every private allocation $\mathbf{x}$ and for every two disjoint coalitions $T_{1}, T_{2} \in \Sigma$ such that $\mu\left(T_{1}\right)>0, \mu\left(T_{2}\right)>0$, and $T_{1} \cup T_{2}=T$, there exists an assignment $\mathbf{y}$ such that $\mathbf{y}(t, \cdot) \in M_{t}$ for almost all $t \in T_{2}$, and such that (A.3.1) $h_{t}(\mathbf{y}(t, \cdot))>h_{t}(\mathbf{x}(t, \cdot))$ for almost all $t \in T_{2}$, and

(A.3.2) for all $\omega \in \Omega: \int_{T_{1}} \mathbf{e}(t, \omega) d \mu+\int_{T_{2}} \mathbf{x}(t, \omega) d \mu \geq \int_{T_{2}} \mathbf{y}(t, \omega) d \mu$.

Condition (A.3), Irreducibility, was introduced in McKenzie (1959) for economies with a finite number of traders, and was extended for atomless economies by Hildenbrand (see Hildenbrand, 1974, pp. 143, 214). It expresses the idea that the endowment of every coalition is desired. Our definition is a variant of Hildenbrand's (1974).

Proposition 3.1. Assume that an economy $\mathscr{E}$ satisfies assumption (A.2). If for almost every $t \in T$ and all $\omega \in \Omega$ we have $\mathbf{e}(t, \omega) \gg 0$, then $\mathscr{E}$ satisfies Condition (A.3) (Irreducibility).

Proof. Assume that $\mathbf{e}(t, \omega) \gg 0$ for almost every $t \in T$ and all $\omega \in \Omega$. Let $\mathbf{x}$ be a private allocation in $\mathscr{E}$, and let $T_{1}, T_{2} \in \Sigma$ be two disjoint coalitions such that $\mu\left(T_{1}\right)>0$ and $\mu\left(T_{2}\right)>0$, and $T_{1} \cup T_{2}=T$. Then for all $\omega \in \Omega$ we have

$$
\int_{T_{1}} \mathbf{e}(t, \omega) d \mu \gg 0 .
$$

Let $a \in \Re_{+}^{l}$ be such that $\mu\left(T_{2}\right) a \gg 0$, and such that for all $\omega \in \Omega$ we have

$$
\int_{T_{1}} \mathbf{e}(t, \omega) d \mu \geq \mu\left(T_{2}\right) a .
$$

Define $\mathbf{y}: T \times \Omega \rightarrow \Re_{+}^{l}$ by

$$
\mathbf{y}(t, \omega)= \begin{cases}0 & t \in T_{1}, \\ \mathbf{x}(t, \omega)+a & t \in T_{2} .\end{cases}
$$

Then for all $t \in T_{2}, \mathbf{y}(t, \cdot) \in M_{t}$. Since for almost all $t \in T$ and all $\omega \in \Omega$, $u_{t}(\omega, \cdot)$ is increasing, we have

$$
h_{t}(\mathbf{y}(t, \cdot))>h_{t}(\mathbf{x}(t, \cdot)),
$$

for almost all $t \in T_{2}$. From the choice of $a$ it is clear that (A.3.2) holds for $\mathbf{x}$ and $\mathbf{y}$.

A quasi equilibrium for the economy $\mathscr{E}$ is a pair $(p, \mathbf{x})$, where $p$ is a price system and $\mathbf{x}$ is a private allocation, such that

(3.5) for almost all $t \in T$, either $\sum_{\omega \in \Omega} p(\omega) \cdot \mathbf{e}(t, \omega)=0$, or the function $\mathbf{x}(t, \cdot)$ maximizes $h_{t}$ on $B_{t}(p)$, and

$$
\sum_{\omega \in \Omega} p(\omega) \cdot \int_{T} \mathbf{x}(t, \omega) d \mu=\sum_{\omega \in \Omega} p(\omega) \cdot \int_{T} \mathbf{e}(t, \omega) d \mu .
$$

Proposition 3.2. If an economy $\mathscr{E}$ satisfies conditions (A.1)- (A.3), then every quasi equilibrium of $\mathscr{E}$ is a competitive equilibrium. 
Proof. Proposition 3.2 is a direct consequence of Proposition 1 in Hildenbrand (1974), p. 214, when for $t \in T$ the consumption sets are $M_{t}$, the utility functions are $h_{t}$, and the production sets are $\left(\Re_{-}^{l}\right)^{\Omega}$.

Theorem A. If an economy $\mathscr{E}$ satisfies assumptions $(A .1)-(A .3)$ then it has a competitive equilibrium.

Proof. First note that our definition of quasi equilibrium is a special case of Hildenbrand's $(1970,1974)$ definition of quasi equilibrium for a coalition production economy where for $t \in T$ the consumption sets are $M_{t}$, the utility functions are $h_{t}$, and the production sets are $\left(\Re_{-}^{l}\right)^{\Omega}$ (see Hildenbrand, 1970, Section 2, p. 611). Therefore by Theorem 2 in Hildenbrand (1970), an economy $\mathscr{E}$ has a quasi equilibrium. Moreover, by Proposition 3.2 any quasi-equilibrium of $\mathscr{E}$ is a competitive equilibrium of $\mathscr{E}$.

The following corollary is a direct consequence of Proposition 3.1 and Theorem A.

Corollary 3.3. If an economy $\mathscr{E}$ satisfies $(A .1),(A .2)$, and in addition for every $\omega \in \Omega$ and almost all $t \in T$ we have $\mathbf{e}(t, \omega) \gg 0$, then $\mathscr{E}$ has a competitive equilibrium.

\section{The private core}

In this section we extend the definition of private core introduced in Yannelis (1991) to our economy, and show that under conditions $(A .1)-(A .3)$ the set of competitive allocations of the economy coincides with the set of private core allocations.

An assignment $\mathbf{x}$ is a private core allocation for the economy $\mathscr{E}$ if (4.1) $\mathbf{x}$ is a private allocation, and

(4.2) there do not exist a coalition $S \in \Sigma$ and an assignment $\mathbf{y}$ such that

(4.2.1) $\mu(S)>0$,

(4.2.2) $\mathbf{y}(t, \cdot)$ is $\mathscr{F}_{t}$-measurable for all $t \in S$,

(4.2.3) $\int_{S} \mathbf{y}(t, \omega) d \mu \leq \int_{S} \mathbf{e}(t, \omega) d \mu$ for all $\omega \in \Omega$, and

(4.2.4) $h_{t}(\mathbf{y}(t, \cdot))>h_{t}(\mathbf{x}(t, \cdot))$ for almost all $t \in S$.

The private core of an economy $\mathscr{E}$ is the set of all private core allocations of $\mathscr{E}$.

Proposition 4.1. Every competitive allocation of an economy $\mathscr{E}$ is a private core allocation of $\mathscr{E}$.

Proof. Proposition 4.1 is a special case of Proposition 2 in Hildenbrand (1974), p. 216. 
Theorem B. Under assumptions (A.1) - (A.3) the set of competitive allocations of an economy $\mathscr{E}$ coincides with the private core of $\mathscr{E}$.

Proof. By Proposition (4.1) it suffices to show that every private core allocation in $\mathscr{E}$ is a competitive allocation. Let $\mathbf{x}$ be a private core allocation in $\mathscr{E}$. By Theorem 1 in Hildenbrand (1974), p. 216, there is a price system $p$ such that $(p, \mathbf{x})$ is a quasi equilibrium for $\mathscr{E}$. By Proposition 3.2 we obtain that $(p, \mathbf{x})$ is a competitive equilibrium for $\mathscr{E} . \square$

The following corollary is a direct consequence of Proposition 3.1 and Theorem B.

Corollary 4.2. If an economy $\mathscr{E}$ satisfies $(A .1),(A .2)$, and in addition for every $\omega \in \Omega$ and almost all $t \in T$ we have $\mathbf{e}(t, \omega) \gg 0$, then the set of competitive allocations of $\mathscr{E}$ coincides with the private core of $\mathscr{E}$.

We now give an example of an atomless "reducible" economy (i.e., it does not satisfy Condition (A.3)) with complete information satisfying (A.1) and (A.2) which has a non-empty core, but does not have a competitive equilibrium. Also this example shows that the private core may not satisfy the Equal Treatment Property when the economy is "reducible."

Example 4.3. Consider an atomless economy $\mathscr{E}$ in which the space of traders is $([0,2], \mathscr{B}, \lambda)$, where $\mathscr{B}$ is the $\sigma$-field of Borel subsets of $[0,2]$ and $\lambda$ is the Lebesgue measure. Traders have complete information, and the commodity space is $\Re_{+}^{2}$. Every trader $t \in T_{1}=[0,1]$ has an initial endowment $\mathbf{e}(t)=$ $(1,0)$ and utility function $u_{1}(x, y)=x$, whereas each trader $t \in T_{2}=(1,2]$ has initial endowment $\mathbf{e}(t)=(1,1)$ and utility function $u_{2}(x, y)=y$. The core of the economy $\mathscr{E}$ consists of all allocations $\mathbf{x}$ such that

$$
u_{t}(\mathbf{x}(t))= \begin{cases}\alpha(t) & t \in T_{1}, \\ 1 & t \in T_{2},\end{cases}
$$

where $\alpha: T_{1} \rightarrow \Re_{+}$is an integrable function such that $\alpha(t) \geq 1$ for almost all $t \in T_{1}$, and $\int_{T_{1}} \alpha(t) d \lambda \leq 2$. It is easy to see that every core allocation in $\mathscr{E}$ is a quasi equilibrium allocation with price system $p=(0,1)$. However, the economy $\mathscr{E}$ does not have a competitive equilibrium. It is worth noticing that the core of this economy contains allocations that do not have the Equal Treatment Property.

In the following example we consider a reducible economy (i.e., it does not satisfy Condition (A.3)) with asymmetric information in which the utility functions of the traders are strictly increasing and strictly concave. The economy does not have a competitive equilibrium, although its private core is non-empty (it consists of the initial assignment).

Example 4.4. Consider an atomless economy $\mathscr{E}$ in which the space of traders is $([0,2], \mathscr{B}, \lambda)$, where $\mathscr{B}$ is the $\sigma$-field of Borel subsets of $[0,2]$ and $\lambda$ is 
the Lebesgue measure. The commodity space is $\Re_{+}^{2}$, and the space of states of nature is $\Omega=\left\{\omega_{1}, \omega_{2}\right\}$. The information partition of every trader $t$ in the interval $T_{1}=[0,1]$ is $\Pi_{1}=\left\{\left\{\omega_{1}\right\},\left\{\omega_{2}\right\}\right\}$, his prior belief is $q_{1}=\left(\frac{1}{2}, \frac{1}{2}\right)$, his initial endowments are $\mathbf{e}\left(t, \omega_{1}\right)=(1,0)$ and $\mathbf{e}\left(t, \omega_{2}\right)=(0,1)$, and his utility functions are $u_{t}(\omega,(x, y))=\sqrt{x}+\sqrt{y}$ for all $\omega \in \Omega$. The information partition of every trader $t$ in the interval $T_{2}=(1,2]$ is $\Pi_{2}=\left\{\left\{\omega_{1}, \omega_{2}\right\}\right\}$, his prior belief is $q_{2}=\left(\frac{1}{2}, \frac{1}{2}\right)$, his initial endowments are $\mathbf{e}\left(t, \omega_{1}\right)=\mathbf{e}\left(t, \omega_{2}\right)=(1,1)$, and his utility function is $u_{t}(\omega,(x, y))=\sqrt{x}+\sqrt{y}$, for all $\omega \in \Omega$. It is easy to see that the economy does not have a competitive equilibrium. However, the unique private core allocation is the initial assignment e. Note that $(p, \mathbf{e})$, where $p\left(\omega_{1}\right)=(0,1)$ and $p\left(\omega_{2}\right)=(1,0)$, is a quasi equilibrium for $\mathscr{E}$.

\section{The weak fine core}

In this section we extend to our model the definition of "weak fine core" introduced by Koutsougeras and Yannelis (1993) (see also Allen, 1991), and we prove an equivalence theorem for this notion of core.

We first note that since $\Omega$ is a finite set, there is a finite number of different information partitions. Let us be given an economy $\mathscr{E}$, and denote by $\Pi_{1}, \ldots, \Pi_{n}$ the $n$ distinct information partitions of the traders. For every $1 \leq i \leq n$, let $\mathscr{F}_{i}$ be the field generated by $\Pi_{i}$, and let

$$
T_{i}=\left\{t \in T \mid \mathscr{F}_{t}=\mathscr{F}_{i}\right\} .
$$

We assume that for every $1 \leq i \leq n$ the set $T_{i}$ is measurable and $\mu\left(T_{i}\right)>0$. If $I \subset\{1, \ldots, n\}$ is a non-empty set, we denote by $\bigvee_{i \in I} \mathscr{F}_{i}$ the smallest field which contains each $\mathscr{F}_{i}, i \in I$. If $S \in \Sigma$ is a coalition with $\mu(S)>0$, we denote

$$
I(S)=\left\{i \mid 1 \leq i \leq n \text { and } \mu\left(S \cap T_{i}\right)>0\right\} .
$$

An assignment $\mathbf{x}$ for the economy $\mathscr{E}$ is called a weak fine core allocation if

(5.1) for almost all $t \in T$ the function $\mathbf{x}(t, \cdot)$ is $\bigvee_{i=1}^{n} \mathscr{F}_{i}$-measurable;

(5.2) for every $\omega \in \Omega, \int_{T} \mathbf{x}(t, \omega) d \mu \leq \int_{T} \mathbf{e}(t, \omega) d \mu$;

(5.3) there do not exist a coalition $S \in \Sigma$ and an assignment $\mathbf{y}$ such that

(5.3.1) $\mu(S)>0$,

(5.3.2) $\mathbf{y}(t, \cdot)$ is $\bigvee_{i \in I(S)} \mathscr{F}_{i}$-measurable for almost all $t \in S$,

(5.3.3) $\int_{S} \mathbf{y}(t, \omega) d \mu \leq \int_{S} \mathbf{e}(t, \omega) d \mu$ for all $\omega \in \Omega$, and

(5.3.4) $h_{t}(\mathbf{y}(t, \cdot))>h_{t}(\mathbf{x}(t, \cdot))$ for almost all $t \in S$.

The weak fine core of $\mathscr{E}$ is defined as the set of all weak fine core allocations of $\mathscr{E}$.

We now introduce the following condition.

(A.4) If $A \in \bigvee_{i=1}^{n} \mathscr{F}_{i}$ is non-empty, then $q_{t}(A)>0$ for almost all $t \in T$.

We denote by $\mathscr{E}^{*}$ an economy identical to $\mathscr{E}$ except for the information fields of the traders, which for all $t \in T$ is taken to be $\mathscr{F}_{t}^{*}=\bigvee_{i=1}^{n} \mathscr{F}_{i}$. Note that the information in $\mathscr{E}^{*}$ is symmetric. 
In the proof of the following proposition we use a result of Vind (1972), see also Proposition 7.3.2. in Mas-Colell (1985), which asserts that in atomless economy (see Aumann, 1964) if an allocation is blocked, then the blocking coalition can be chosen with a measure which is arbitrarily close to the measure of the grand coalition.

Proposition 5.1. Assume that an economy $\mathscr{E}$ satisfies (A.1), (A.2) and (A.4), and in addition for almost all $t \in T$ and for every $\omega \in \Omega$ the function $u_{t}(\omega, \cdot)$ is strictly increasing. Then the weak fine core of $\mathscr{E}$ coincides with the private core of $\mathscr{E}^{*}$.

Proof. It is clear that every private core allocation in $\mathscr{E}^{*}$ is a weak fine core allocation of $\mathscr{E}$. We prove the converse. Let $\Pi=\bigvee_{i=1}^{n} \Pi_{i}$ (i.e., $\Pi$ is the smallest partition of $\Omega$ that refines each $\Pi_{i}$ ). Denote $\Pi=\left\{A_{1}, \ldots, A_{k}\right\}$, and let $X$ be the set of all members of $\left(\Re_{+}^{l}\right)^{\Omega}$ which are $\bigvee_{i=1}^{n} \mathscr{F}_{i}$-measurable. Then every member of $X$ is constant on every $A_{j}, 1 \leq j \leq k$. Let the function $\alpha: X \rightarrow \Re_{+}^{k l}$ be defined by $\alpha(x)=\hat{x}$, where for $1 \leq j \leq k, \hat{x}_{j}=x\left(\omega_{j}\right)$ for some $\omega_{j} \in A_{j}$. Note that $\alpha$ is a one to one mapping from $X$ onto $\Re_{+}^{k l}$. For every $t \in T$ we define a function $\hat{h}_{t}: \Re_{+}^{k l} \rightarrow \Re$ by $\hat{h}_{t}(\hat{x})=h_{t}\left(\alpha^{-1}(\hat{x})\right)$. Then $\hat{h}_{t}$ is continuous, and by $(A .4)$ it is strictly increasing. Consider now the complete information atomless economy $\widehat{\mathscr{E}}$ in which the space of traders is $(T, \Sigma, \mu)$, the commodity space is $\Re_{+}^{k l}$, the initial assignment is $\hat{\mathbf{e}}$, where $\hat{\mathbf{e}}(t)=\alpha(\mathbf{e}(t, \cdot))$ for all $t \in T$, and the utility function of trader $t$ is $\hat{h}_{t}$. Let $\mathbf{y}$ be a weak fine core allocation of $\mathscr{E}$. Assume, contrary to our claim, that $\mathbf{y}$ is not a private core allocation of $\mathscr{E}^{*}$. For every $t \in T$ let $\hat{\mathbf{y}}(t)=\alpha(\mathbf{y}(t, \cdot))$. Then $\hat{\mathbf{y}}$ is not in the core of the economy $\hat{\mathscr{E}}$. Therefore by the Theorem of Vind (1972), there exists a coalition $S \in \Sigma$ and an assignment $\hat{\mathbf{z}}$ in $\widehat{\mathscr{E}}$ such that $\mu(S)>\mu(T)-\min \left\{\mu\left(T_{1}\right), \ldots, \mu\left(T_{n}\right)\right\}, \int_{S} \hat{\mathbf{z}}(t) d \mu \leq \int_{S} \hat{\mathbf{e}}(t) d \mu$, and $\hat{h}_{t}(\hat{\mathbf{z}}(t))>\hat{h}_{t}(\hat{\mathbf{y}}(t))$ for almost all $t \in S$. For every $t \in T$ let $\mathbf{z}(t, \cdot)=\alpha^{-1}(\hat{\mathbf{z}}(t))$. Then for every $\omega \in \Omega$ we have

$$
\int_{S} \mathbf{z}(t, \omega) d \mu \leq \int_{S} \mathbf{e}(t, \omega) d \mu .
$$

Since $\mu(S)>\mu(T)-\min \left\{\mu\left(T_{1}\right), \ldots, \mu\left(T_{n}\right)\right\}$, we have $I(S)=\{1,2, \ldots, n\}$ and thus for all $t \in T, \mathbf{z}(t, \cdot)$ is $\bigvee_{i \in I(S)} \mathscr{F}_{i}$-measurable.

For almost all $t \in S$ we have

$$
h_{t}(\mathbf{z}(t, \cdot))=\hat{h}_{t}(\hat{\mathbf{z}}(t))>\hat{h}_{t}(\hat{\mathbf{y}}(t))=h_{t}(\mathbf{y}(t, \cdot)) \text {, }
$$

which contradicts the assumption that $\mathbf{y}$ is a weak fine core allocation of $\mathscr{E}$.

Lemma 5.2. Assume that an economy $\mathscr{E}$ satisfies the assumptions of Proposition 5.1, and in addition for almost every $t \in T$ there is $\omega \in \Omega$ such that $\mathbf{e}(t, \omega) \neq 0$. Then the economy $\mathscr{E}^{*}$ is irreducible, i.e., it satisfies condition (A.3). 
Proof. Let $\mathbf{x}$ be a private allocation in $\mathscr{E}^{*}$, and let $T_{1}, T_{2}$ be two disjoint coalitions in $\Sigma$ such that $T=T_{1} \cup T_{2}$, and $\mu\left(T_{1}\right)>0, \mu\left(T_{2}\right)>0$. For every $(t, \omega) \in T \times \Omega$ let

$$
\mathbf{y}(t, \omega)= \begin{cases}0 & t \in T_{1}, \\ \mathbf{x}(t, \omega)+\frac{1}{\mu\left(T_{2}\right)} \int_{T_{1}} \mathbf{e}(t, \omega) d \mu & t \in T_{2} .\end{cases}
$$

Then for every $t \in T, \mathbf{y}(t, \cdot)$ is $\bigvee_{i=1}^{n} \mathscr{F}_{i}$-measurable. Since $u_{t}(\omega, \cdot)$ is strictly increasing for almost all $t \in T$ and all $\omega \in \Omega$, and $\frac{1}{\mu\left(T_{2}\right)} \int_{T_{1}} \mathbf{e}(t, \omega) d \mu>0$ for some $\omega \in \Omega$, it follows from (A.4) that for almost every $t \in T_{2}$

$$
h_{t}(\mathbf{y}(t, \cdot))>h_{t}(\mathbf{x}(t, \cdot)) .
$$

Moreover, for all $\omega \in \Omega$ we have

$$
\int_{T_{1}} \mathbf{e}(t, \omega) d \mu+\int_{T_{2}} \mathbf{x}(t, \omega) d \mu=\int_{T_{2}} \mathbf{y}(t, \omega) d \mu .
$$

Therefore $\mathscr{E}^{*}$ is irreducible.

Theorem C. Assume that an economy $\mathscr{E}$ satisfies the assumptions of Lemma 5.2. Then the weak fine core of $\mathscr{E}$ coincides with the set of competitive allocations of $\mathscr{E}^{*}$.

Proof. The proof follows directly from Proposition 5.1, Lemma 5.2 and Theorem B.

We conclude with the following proposition.

Proposition 5.3. If an economy $\mathscr{E}$ satisfies the assumptions $(A .1),(A .2)$, and in addition for every $\omega \in \Omega$ and almost all $t \in T, \mathbf{e}(t, \omega) \gg 0$, then the weak fine core of $\mathscr{E}$ coincides with the set of competitive allocations of $\mathscr{E}^{*}$.

Proof. The proof is the same as that of Theorem C, noticing that $\mathscr{E}^{*}$ is irreducible and the Theorem of Vind (1972) holds under the assumptions of Proposition 5.3.

\section{References}

Allen, B : Market games with asymmetric information and non-transferable utility: representations results, and the core Mimeo, University of Pennsylvania (1991)

Aumann, R J : Markets with a continuum of traders Econometrica 32, 39-50 (1964)

Aumann, R J : Existence of competitive equilibria in markets with a continuum of traders Econometrica 34, 1-17 (1966)

Einy, E, Shitovitz, B : Private value allocations in large economies with differential information Games and Economic Behavior (forthcoming) (1999)

Einy, E, Moreno, D, Shitovitz, B : The bargaining set of a large economy with differential information Economic Theory (forthcoming) (1999)

Hildenbrand, W : Existence of equilibria for economies with production and a measure space of economic agents Econometrica 34, 1-17 (1970) 
Hildenbrand, W.: Core and equilibria in large economies. Princeton: Princeton University Press 1974 Koutsougeras, L.C., Yannelis, N.C.: Incentive compatibility and information superiority of the core of an economy with differential information. Economic Theory 3, 195-216 (1993)

Mas-Colell, A.: The theory of general equilibrium: a differentiable approach. Cambridge, MA: Cambridge University Press 1985

McKenzie, L.W.: On the existence of general equilibrium for a competitive market. Econometrica 27, 54-71 (1959)

Radner, R.: Competitive equilibrium under uncertainty. Econometrica 36, 31-58 (1968)

Radner, R.: Equilibrium under uncertainty. In: K.J. Arrow, Intriligator, M.D. (eds.) Handbook of mathematical economics, vol. II. Amsterdam: North Holland 1982

Vind, K.: A third remark on the core of an atomless economy. Econometrica 40, 585-586 (1972)

Wilson, R.: Information, efficiency, and the core of an economy. Econometrica 46, 807-816 (1978)

Yannelis, N.C.: The core of an economy with differential information. Economic Theory 1, 183-198 (1991) 